\title{
SOME FOURIER-STIELTJES COEFFICIENTS REVISITED
}

\author{
SYLVESTER REESE
}

(Communicated by J. Marshall Ash)

\begin{abstract}
We give a new proof of a result of R. Salem: The Fourier-Stieltjes coefficients of certain strictly increasing singular functions do not vanish at infinity.
\end{abstract}

Let $p, q$ be two fixed positive numbers such that $p+q=1$ and $p \neq q$. Let the number $x$ in the left-closed unit interval be represented by its dyadic expansion: $x=\sum_{l}^{\infty} d_{n} 2^{-n}$, where $d_{n}=0$ or 1 ; in the ambiguous case of the dyadic rationals, choose the finite expansion. Let the function $H=H_{p}$ be defined in the closed unit interval $l$ by

$$
H(1)=1 ; H(x)=\sum_{l}^{\infty} d_{n} p^{n-(s(n)-1)} q^{s(n)-1},
$$

$x$ in $[0,1)$, and $s(n)=\sum_{l}^{n} d_{k}$. Then Salem [14] proved that $H$ is a strictly increasing singular continuous function on $l$ and that its Fourier-Stieltjes coefficients do not vanish at infinity; he demonstrated this latter property of $H$ by calculating the coefficients via approximating Riemann-Stieltjes sums. In this note we give a proof of this property by exploiting the self-similarity of the function $H$.

It is easy to see that $H$ satisfies the self-similar functional equations:

$$
\begin{aligned}
H(x / 2) & =p H(x) \\
H((1+x) / 2) & =p+q H(x) \quad 0 \leq x \leq 1 .
\end{aligned}
$$

Consider now the Fourier-Stieltjes coefficients of $H$ defined by

$$
c_{n}=c_{n}(d H)=\int_{0}^{l} \exp (-i 2 \pi n x) d H(x),
$$

where $n=0, \pm 1, \pm 2, \ldots$; the integrals are Riemann-Stieltjes integrals.

Received by the editors May 3, 1988. Presented at the Centennial Celebration of the AMS in Providence, RI, on August 9, 1988 (844-42-81).

1980 Mathematics Subject Classification (1985 Revision). Primary 42A16, 42A38, 26A30; Secondary 39B20, 60G40.

Key words and phrases. Fourier-Stieltjes coefficient, singular function. 
Theorem. The Fourier-Stieltjes coefficients of $H$ do not vanish at infinity. Proof. Consider $c_{n}(d H)$ and set $n=2^{m}$, where $m>0$. Then

$$
c_{2^{m}}=\int_{0}^{l} \exp \left(-i \pi 2^{m+1} x\right) d H(x) \text {. }
$$

Splitting the integral into two integrals-from 0 to $\frac{1}{2}$ and $\frac{1}{2}$ to 1 -and substituting suitably, we have $c_{2^{m}}$ equals

$$
\int_{0}^{l} e^{-i \pi 2^{m} x} d H(x / 2)+\int_{0}^{l} e^{-i \pi 2^{m}(1+x)} d H((1+x) / 2)
$$

Using now the self-similar equations (1), $c_{2^{m}}$ becomes

$$
\int_{0}^{l} \exp \left(-i \pi 2^{m} x\right) p d H(x)+\int_{0}^{l} \exp \left(-i \pi 2^{m} x\right) q d H(x) .
$$

Combining these integrals, we have $c_{2^{m}}=c_{2^{m-1}}$. Thus $c_{2^{m}}=c_{1}$ for all nonnegative integers $m$. If we can show that $c_{1} \neq 0$, then we will be done.

Consider now the imaginary part of $c_{1}$ :

$$
\operatorname{Im}\left(c_{1}\right)=-\int_{0}^{l} \sin (2 \pi x) d H(x) .
$$

Using the same steps as before-splitting the integral into two parts (from 0 to $\frac{1}{2}$ and $\frac{1}{2}$ to 1 ), substituting suitably, and using the self-similar equations (1) -we have

$$
\operatorname{Im}\left(c_{1}\right)=(q-p) \int_{0}^{l} \sin (\pi x) d H(x) \neq 0 .
$$

The last inequality follows from: $p \neq q ; \sin (\pi x)$ is positive and $H$ is strictly increasing for $0<x<1$. Hence, $c_{1} \neq 0$.

Remarks 1. This proof was suggested by Hardy and Rogosinski's use [6, Theorem 41, p. 27] of the self-similarity of Cantor's singular function to show that its Fourier-Stieltjes coefficients do not vanish at infinity. Note, however, that there are uncountably many "self-similar Cantor-like" singular continuous functions on $l$ whose Fourier-Stieltjes coefficients do vanish at infinity. See Salem [15] or Zygmund [17, Volume 2, Theorem 11.16]. Note also that there are uncountably many $H_{p}$ 's on the interval $l$.

2. An equivalent form of the functional equations (1) play a central role in Dubins and Savage's $[4$, p. 85$]$ gambling strategy called BOLD play.

3. In [12], de Rham used the functional equations (1) to define the functions $H_{p}$.

4. Lastly, we note that the functions $H_{p}$ have been appearing in the literature in various forms since at least 1906 . They gained a wide exposure in the wellknown book of Riesz and Sz.-Nagy [13, p. 48]. Besides those authors already cited, see Cesàro [3], Hellinger [7, p. 27], Faber [5, p. 395], Lomnicki and Ulam [11, p. 268], Kakutani [9], Hewitt and Stromberg [8, p. 278], Billingsley [1, p. $36 ; 2$, pp. 85, 361], Takacs [16], and Laczkovich [10]. The books of Billingsley have some graphs of $H_{p}$ for some specific $p$ 's. 


\section{REFERENCES}

1. P. Billingsley, Ergodic theory and information, John Wiley \& Sons, New York, 1965.

2. __ Probability and measure, John Wiley \& Sons, New York, 1979.

3. E. Cesàro, Functions continues sans dérivée, Arch. Math. Phys. 10 (1906), 57-63.

4. L. E. Dubins and L. J. Savage, Inequalities for stochastic processes, reprinted, Dover Publications, New York, 1976. (1st. eds., How to gamble if you must, McGraw-Hill Book Co., New York, 1965.)

5. G. Faber, Über stetige Funktionen II, Math. Ann. 69 (1910), 372-443.

6. G. H. Hardy and W. W. Rogosinski, Fourier series, 3rd ed., Cambridge University Press, New York, 1965.

7. E. Hellinger, Die Orthogonalinvarianten quadratischer Formen von unendlichvielen Variabelen, Dissertation, Göttingen, 1907.

8. E. Hewitt and K. Stromberg, Real and abstract analysis, Springer-Verlag, New York, 1965.

9. S. Kakutani, On equivalence of infinite product measures, Ann. Math. 49 (1948), 214-224.

10. M. Laczkovich, Some remarks on Faber's singular function, Mat. Lapok 30 (1978/82), 69-79.

11. Z. Lomnicki et S. Ulam, Sur la théorie de la mesure dans les espaces combinatoires et son application au calcul des probabilités I. Variables indépendantes, Fund. Math. 23 (1934), 237278.

12. G. de Rham, Sur quelques courbes définies par des équations fonctionnnelles, Rend. Sem. Mat. Univ. Politec. Torino 16 (1957), 101-113. (Reprinted in G. de Rham, Oeuvres mathématiques, Univ. de Gen., Enseign. Math., 1981 pp. 716-728.

13. F. Riesz et B. Sz.-Nagy, Leçons d'analyse fonctionnelle, Akadémiai Kiadó, Budapest, 1952.

14. R. Salem, On some singular monotonic functions which are strictly increasing, Trans. Amer. Math. Soc. 53 (1943), 427-439.

15. __ Sets of uniqueness and sets of multiplicity, Trans. Amer. Math. Soc. 54 (1943), 218-28.

16. L. Takács, An increasing continuous singular function, Amer. Math. Monthly 85 (1978), 35-37.

17. A. Zygmund, Trigonometric series, 2 vols, Cambridge University Press, New York, 1959.

Queensborough Community College, Bayside, New York 11364 